\title{
Effect of Prolonged Phthalate Ester Administration on Rat Liver *
}

\author{
A.E. GANNING, ${ }^{\mathrm{a}} \AA$ A. ELHAMMER, ${ }^{\mathrm{a}}$ U. BRUNK, ${ }^{\mathrm{b}}$ and G. DALLNER ${ }^{\mathrm{c}}$ \\ ${ }^{a}$ Department of Biochemistry, University of Stockholm, S-106 91 Stockholm, \\ b Department of Pathology, University of Linköping, S-581 85 Linköping and \\ c Department of Pathology, Karolinska Institutet, S-104 01 Stockholm, Sweden
}

Phthalate esters which are used as plasticizers for PVC plastics are present in large amounts in our environment. The acute toxicity of these substances is very low but a considerable amount of available data indicates significant chronic toxicity. ${ }^{1}$ In vitro tests proved to be ineffective for the assessment of phthalate toxicity; instead large doses of plasticizers were supplied during a relatively short time period. ${ }^{2}$ Our aim with the investigation was to study the mechanisms of induction of peroxisomes and mitochondria in the liver and also to create experimental conditions similar to those occurring in man.

Male rats weighing $180 \mathrm{~g}$ at the start of the experiments were used. Various concentrations of di(2-ethylhexyl)phthalate (DEHP) were added to the diet which was given ad libitum. Since parts of the experiments were conducted over a two year period, great care was taken to keep a high standard of the animal quarters.

Treatment of rats with DEHP results in induction of peroxisomes and mitochondria; a number of enzymes are also induced at various locations. ${ }^{3}$ In vivo experiments were performed to find out the incorporation rate of amino acid precursors into the total protein. In the initial phase of phthalate induction, a substantial increase in the incorporation rate could be observed both into the mitochondrial and microsomal proteins. However, it seemed questionable that the extensive induction taking place already during the first week could be explained only by one mechanism. For this reason, breakdown rates of total proteins in the mitochondria, microsomes and particle-free supernatant were determined. Rats received $\left[{ }^{35} \mathrm{~S}\right]$ methionine and at various time-points they were sacrificed and fractions of the liver homogenate were prepared (Fig. 1). The results clearly demonstrate that DEHP interferes with membrane protein turnover and changes the breakdown rate. The half-life of proteins in all fractions increased but the extent of this increase varied. In the controls, the half-life of mitochondrial proteins is $\sim 6 \mathrm{~d}$ which after treatment changes to $\sim 22 \mathrm{~d}$. Therefore, it appears that the decreased breakdown is a contributing factor in mitochondrial proliferation. The change in half-life for microsomes and supernatant occurs to a more limited extent; for the microsomes it is $\sim 3.5$ to $\sim 5.5 \mathrm{~d}$ and for the supernatant from $\sim 2.5$ to $\sim 5 \mathrm{~d}$. Obviously, both increased synthesis and decreased breakdown play roles in the induction mechanism leading to the appearance of new intracellular structures.

For long-term experiments, three different DEHP concentrations were applied: 2, 0.2 and $0.02 \%$. The latter concentration is within a range that is applicable to human conditions. The peroxisomal $\beta$-oxidation of fatty acids is induced extensively during plasticizer treatment. Therefore, one enzyme of this cycle, the $\mathrm{KCN}$-insensitive palmitoylCoA dehydrogenase activity, was followed (Fig. 2). When using the highest DEHP concentration in the diet, the enzyme is rapidly induced about 20 times during 20 weeks. Applying a dose that is ten times lower, the rate of induction is slower but the enzyme activity still increases continuously. In spite of this increase, the activity is not yet at its maximum after two years. A slow but continuous elevation of activity was also obtained when the $0.02 \%$ DEHP concentration was supplied in the diet.

Among the mitochondrial enzymes, those participating in fatty acid transport were induced most extensively during the plasticizer treatment. The carnitine-acetyl transferase activity displays a very rapid induction with $2 \%$ DEHP, already in the first few weeks. (Fig. $3 A)$. Similar to the $\beta$-oxidation enzymes in the peroxisomes, the two lower doses give a much slower induction but again the increase in activity is continuous. The microsomal enzymes measured, such as NADH- and NADPH-cytochrome $c$ reductases, cytochrome $b_{5}$,

\footnotetext{
* Communication at the Meetings of the Swedish Biochemical Society in Stockholm, 24-25th August, 1984.
} 

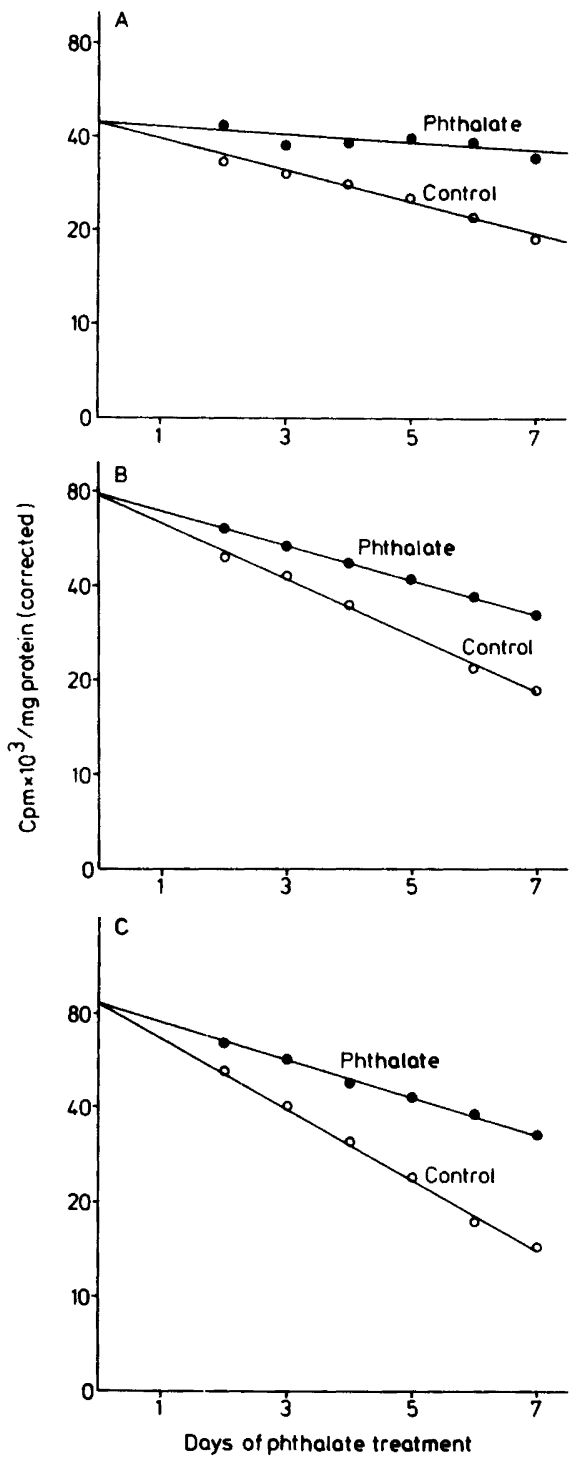

Fig. 1. Changes in the apparent half-lives of intracellular proteins caused by DEHPtreatment. Rats fed a diet containing $2 \%$ DEHP for two days prior to the experiment were given $19 \mathrm{MBq}\left[{ }^{35}\right.$ S]methionine intraperitoneally. Subsequently, the rats were sacrificed at different specified hours after the injection. Mitochondria (A), microsomes (B) and particle-free supernatant (C) were prepared. The protein-bound radioactivity in the individual fractions was determined after trichloroacetic acid precipitation.

glucose-6-phosphatase and ATPase, were influenced only to a limited extent or not at all. The cytochrome P-450 system is induced particularly in the initial phase, but the effect can only be observed with the highest dose (Fig. 3B).

A number of enzyme systems associated with various intracellular membranes were also measured; many of them exhibited only small changes in comparison with the controls. DEHP has a pronounced but selective effect on cellular structure and metabolism.

Thus, the results demonstrate that an extensive induction of hepatic peroxisomes and mitochondria during DEHP treatment is a complex process elicited by both increased synthesis and decreased breakdown of macromolecules. This explains why the induction is so rapid. The long-term experiments indicated that some kind of accumulation of either DEHP or its metabolites occured since the effects even with low doses increased continuously. Consequently, it is incorrect to speak about threshold values for phthalate 


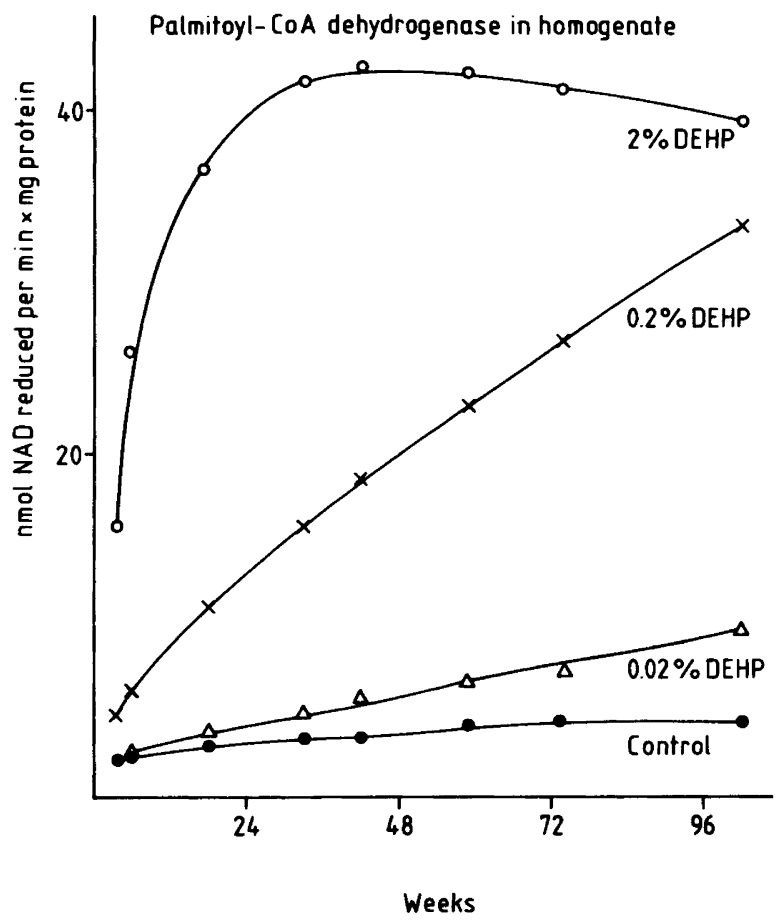

Fig. 2. Palmitoyl-CoA dehydrogenase activity of the liver homogenate. The enzyme activity was measured in the presence of $\mathrm{KCN}^{4}$

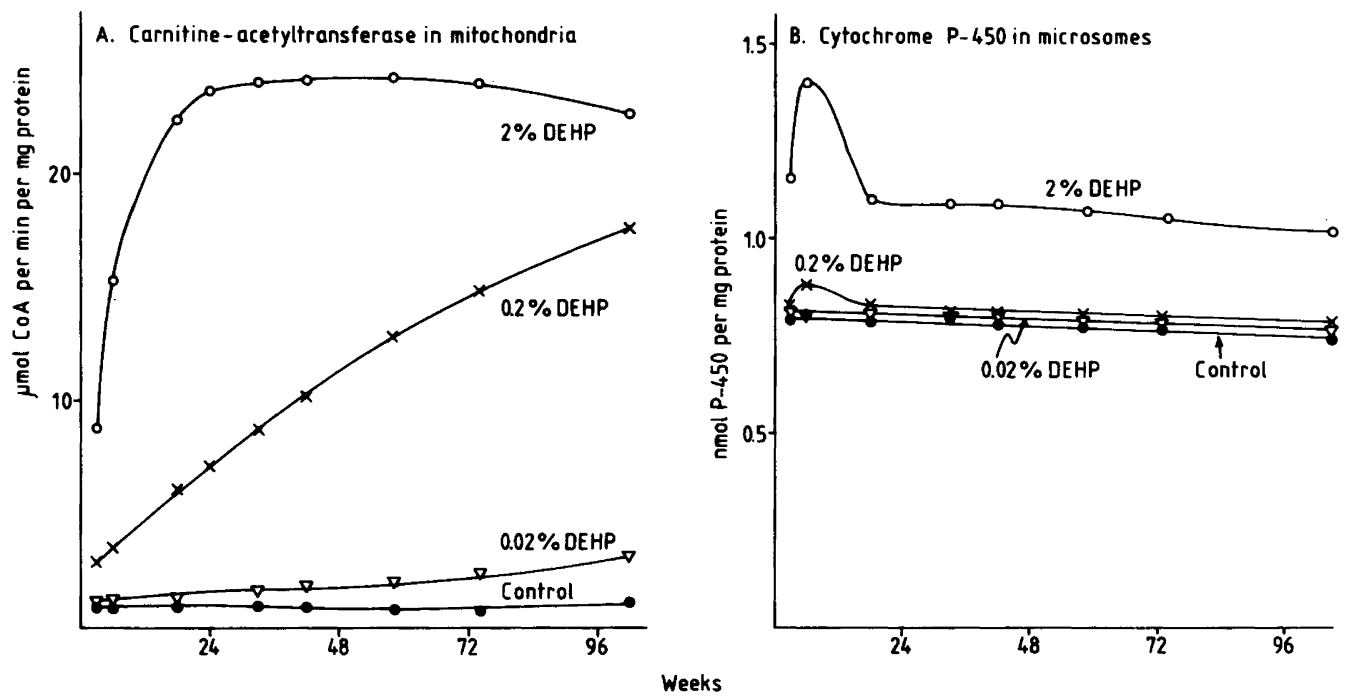

Fig. 3. Effect of prolonged phthalate ester treatment on mitochondria and microsomes. A. Carnitine-acetyl transferase activity in isolated mitochondria. Measurements were performed as earlier. ${ }^{5}$ B. Cytochrome P-450 in isolated microsomes. The amount was estimated with difference spectroscopy. ${ }^{6}$

Acta Chem. Scand. B 39 (1985) No. 4 
esters since all intake can, after a sufficiently long period, give pronounced biological effects.

Acknowledgements. This work was supported by the Swedish Council for Planning and Coordination of Research and the Swedish Medical Research Council.

1. Thomas, J.A., Darby, T.D., Wallin, R.F., Garvin, P.J. and Martis, L. Toxicol. Appl. Pharmacol. 45 (1978) 1.

2. Simmon, V.F., Kauhanen, K. and Tardiff, R.G. In Scott, D., Bridges, B.A. and Sobels, F.M., Eds., Progress in Genetic Toxicology, Elsevier, New York 1977, p. 249.

3. Ganning, A.E., Brunk, U. and Dallner, G. Biochim. Biophys. Acta 763 (1983) 72.

4. Lazarow, P.B. and De Duve, C. Proc. Natl. Acad. Sci. U.S.A. 73 (1976) 2043.

5. Bieber, L.L., Abraham, T. and Helmrath, T. Anal. Biochem. 50 (1972) 509.

6. Omura, T. and Sato, R. J. Biol. Chem. 239 (1964) 2370.

Received September 20, 1984. 